PHYSICAL REVIEW D 93, 109903(E) (2016)

\title{
Erratum: Glueball decay rates in the Witten-Sakai-Sugimoto model [Phys. Rev. D 91, 106002 (2015)]
}

Frederic Brünner, Denis Parganlija, and Anton Rebhan

(Received 26 April 2016; published 18 May 2016)

DOI: $10.1103 /$ PhysRevD.93.109903

The entries for Table IX with tensor glueball mass extrapolated to $M=2000$ and $2400 \mathrm{MeV}$ were incomplete. The corrected table reads:

TABLE IX. Extrapolation of tensor glueball decay for the case of massive pseudo-Goldstone bosons with glueball mass $M=M_{T}=M_{D}$ and when the latter is raised to $2 \mathrm{GeV}$ or the lattice prediction $\sim 2.4 \mathrm{GeV}$. The 't Hooft coupling is again varied from 16.63 to 12.55 .

\begin{tabular}{lll}
\hline \hline Decay & $M$ & $\Gamma / M[T(M)]$ \\
\hline$T \rightarrow 2 \pi$ & 1487 & $0.013 \ldots 0.018$ \\
$T \rightarrow 2 K$ & 1487 & $0.004 \ldots 0.006$ \\
$T \rightarrow 2 \eta$ & 1487 & $0.0005 \ldots 0.0007$ \\
$T$ (total) & 1487 & $\approx 0.02 \ldots 0.03$ \\
\hline$T \rightarrow 2 \rho \rightarrow 4 \pi$ & 2000 & $0.135 \ldots 0.178$ \\
$T \rightarrow 2 K^{*} \rightarrow 2(K \pi)$ & 2000 & $0.119 \ldots 0.177$ \\
$T \rightarrow 2 \omega \rightarrow 6 \pi$ & 2000 & $0.045 \ldots 0.059$ \\
$T \rightarrow 2 \pi$ & 2000 & $0.014 \ldots 0.018$ \\
$T \rightarrow 2 K$ & 2000 & $0.010 \ldots 0.013$ \\
$T \rightarrow 2 \eta$ & 2000 & $0.0018 \ldots 0.0024$ \\
$T$ (total) & 2000 & $\approx 0.32 \ldots 0.45$ \\
\hline$T \rightarrow 2 K^{*} \rightarrow 2(K \pi)$ & 2400 & $0.173 \ldots 0.250$ \\
$T \rightarrow 2 \rho \rightarrow 4 \pi$ & 2400 & $0.159 \ldots 0.211$ \\
$T \rightarrow 2 \omega \rightarrow 6 \pi$ & 2400 & $0.053 \ldots 0.070$ \\
$T \rightarrow 2 \phi$ & 2400 & $0.032 \ldots 0.051$ \\
$T \rightarrow 2 \pi$ & 2400 & $0.014 \ldots 0.019$ \\
$T \rightarrow 2 K$ & 2400 & $0.012 \ldots 0.016$ \\
$T \rightarrow 2 \eta$ & 2400 & $0.0025 \ldots 0.0034$ \\
$T$ (total) & 2400 & $\approx 0.45 \ldots 0.62$ \\
\hline \hline
\end{tabular}

For the decays into vector mesons $K^{*}$ and $\phi$ we have taken into account that their masses are larger than $m_{\rho}$ in the phase space factor, but we have left open the possibility that this also increases the coupling $t_{2} \equiv \sqrt{6} d_{2}$ associated with the vector meson mass term and merged the two alternatives ( $t_{2}$ unchanged and $t_{2}$ multiplied by $m_{K^{*}, \phi}^{2} / m_{\rho}^{2}$ ) in the range of results for the corresponding decay rates.

There is no change to our conclusion that in the Witten-Sakai-Sugimoto model a tensor glueball extrapolated to a mass of $2.4 \mathrm{GeV}$ has a width (now 1.1 to $1.5 \mathrm{GeV}$ ) which is much broader than that of all established $f_{2}$ mesons. With a mass around $2 \mathrm{GeV}$, the corrected width (600 to $900 \mathrm{MeV}$ ) turns out to be larger but perhaps marginally comparable with that of the tensor glueball candidate $f_{2}(1950)$, which has $\Gamma=472(18) \mathrm{MeV}$. 